\title{
Promoter-Induced Reactive Phase Separation in Surface Reactions
}

\author{
Y. De Decker, ${ }^{1}$ H. Marbach, ${ }^{2}$ M. Hinz, ${ }^{2}$ S. Günther, ${ }^{2}$ M. Kiskinova, ${ }^{3}$ A. S. Mikhailov, ${ }^{4}$ and R. Imbihl ${ }^{2}$ \\ ${ }^{1}$ Center for Nonlinear Phenomena and Complex Systems, Université Libre de Bruxelles, Campus Plaine, \\ C.P. 231. B-1050 Brussels, Belgium \\ ${ }^{2}$ Institut für Physikalische Chemie und Elektrochemie, Hannover, Hannover, Germany \\ ${ }^{3}$ Sincrotrone Trieste, Area Science Park-Basovizza, I-34012 Trieste, Italy \\ ${ }^{4}$ Abteilung Physikalische Chemie, Fritz-Haber-Institut der Max-Planck-Gesellschaft, Faradayweg 4-6, 14195 Berlin, Germany
} (Received 15 December 2003; published 14 May 2004)

\begin{abstract}
Promoters are adsorbed mobile species which do not directly participate in a catalytic surface reaction, but can influence its rate. Often, they are characterized by strong attractive interactions with one of the reactants. We show that these conditions lead to a Turing instability of the uniform state and to the formation of reaction-induced periodic concentration patterns. Experimentally such patterns are observed in catalytic water formation on a $\mathrm{Rh}(110)$ surface in the presence of coadsorbed potassium.
\end{abstract}

DOI: 10.1103/PhysRevLett.92.198305

Reactive condensed matter may exhibit nonequilibrium pattern formation resulting from an interplay between reactions, diffusion, and energetic interactions between the reacting particles. Adding a reaction to a phase-separating polymer mixture generates periodic stationary patterns whose wavelength is directly controlled by the reaction rate [1-3]. Phase-separating two-component Langmuir monolayers with chemical reactions show spontaneous formation of traveling wave patterns $[4,5]$. Catalytic surface reactions provide another important example of such systems. Energetic interactions between adsorbed atoms or molecules often lead to two-dimensionally ordered adsorbate phases and structural phase transitions [6]. The theoretical analysis has shown that, with the additional presence of reaction and diffusion, stationary and traveling nonequilibrium patterns based on reactive phase separation can develop in such adsorbate layers [7-9]. In this Letter, we provide evidence that reactive phase separation may occur in a broad class of catalytic surface reactions involving promoters and poisons. Such phase separation is demonstrated experimentally with the catalytic surface reaction $\mathrm{O}_{2}+\mathrm{H}_{2}$ on a potassium-covered $\mathrm{Rh}(110)$ surface. Theoretically, we show that already a general model of a binary annihilation reaction in presence of a promoter species leads to such phenomena. Using a realistic model of the reaction, the experimental results can even be quantitatively reproduced.

Promoters and poisons are substances which, adsorbed on a catalytic metal surface, can dramatically increase or decrease the rate of a catalytic reaction by their mere presence [10]. Alkali metals are a well-known example of a promoter species finding use in a number of technologically important catalytic reactions $[10,11]$. The specific system considered here is the $\mathrm{O}_{2}+\mathrm{H}_{2}$ reaction on a potassium-covered $\mathrm{Rh}(110)$ surface. Both gases, $\mathrm{O}_{2}$ and $\mathrm{H}_{2}$, adsorb dissociatively and the atomic adsorbates then recombine forming water which rapidly desorbs [12]. The alkali metal represents an additional species that modifies
PACS numbers: $82.40 . \mathrm{Np}$, 68.43.Jk, 82.40.-g, 82.65.+r

the local catalytic activity but is not consumed by the reaction. It is very mobile on the oxygen free surface and has a high affinity to one of the reactants, namely, oxygen, with which it forms a number of well-ordered twodimensional coadsorbate phases [13]. In order to visualize the reaction dynamics we employ photoemission electron microscopy (PEEM) which images the local work function. Direct chemical information is provided by scanning photoelectron microscopy (SPEM) - a technique that utilizes synchrotron radiation $(600 \mathrm{eV})$ to eject photoelectrons from element-specific core levels [14]. It has been demonstrated in our previous experiments that reaction fronts initiated under reaction conditions not only remove chemisorbed oxygen but also induce a redistribution of the alkali metal leading to the formation of large $\mathrm{K}+\mathrm{O}$ islands [15-17].

In this Letter, we show that the spatially uniform state in this reaction is unstable and stationary patterns with a characteristic wavelength are spontaneously developing. In contrast to previous experiments, where the initial state of an oxygen-covered surface resulted in low mobility of potassium and, as a consequence, irregular structures, we start here from reducing conditions and a uniform initial state. Decreasing $p_{\mathrm{H}_{2}}$ below a critical value, we observe the spontaneous development of a stationary pattern, as demonstrated by a sequence of PEEM images in Fig. 1(a). The bright area in these images can be attributed to potassium which is known to strongly lower the work function of transition metals [10]. A better ordering of the pattern can be achieved at higher temperature and the corresponding image in Fig. 1(b) already exhibits a clear periodicity in the pattern with an intrinsic wavelength of approximately $50 \mu \mathrm{m}$. Concentration profiles of a stationary pattern obtained from in situ elemental mapping with SPEM are reproduced in Fig. 2. A high coverage coadsorption phase of oxygen and potassium is present while the surrounding area is largely depleted of both adsorbates. The high affinity between potassium and oxygen is apparently the main driving force for the 


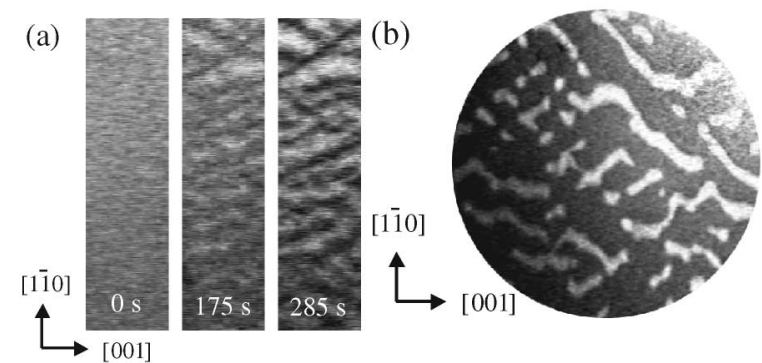

FIG. 1. Formation of a stationary concentration pattern in catalytic water formation on a $\mathrm{Rh}(110)$ surface predosed with potassium $\left(\theta_{\mathrm{K}}=0.08\right)$. (a) PEEM images showing the development of stationary patterns during the transition from reducing to oxidizing conditions. Experimental conditions: $T=518 \mathrm{~K}$, $p_{\mathrm{H}_{2}}=2.2 \times 10^{-6} \mathrm{mbar}, p_{\mathrm{O}_{2}}=1 \times 10^{-6} \operatorname{mbar}(t=0), 1.5 \times$ $10^{-6} \operatorname{mbar}(t=175 \mathrm{~s}), 2 \times 10^{-6} \operatorname{mbar}(t=285 \mathrm{~s})$. The dimensions of imaged area are $210 \times 35 \mu \mathrm{m}$. (b) PEEM image of a stationary concentration pattern recorded $840 \mathrm{~s}$ after adjusting reaction conditions. Experimental conditions: $T=565 \mathrm{~K}$, $p_{\mathrm{O}_{2}}=2 \times 10^{-7}$ mbar, $p_{\mathrm{H}_{2}}=1.8 \times 10^{-7}$ mbar. The diameter of the imaged area is $400 \mu \mathrm{m}$.

condensation process. Both hydrogen and potassium, are highly mobile and one can suspect that the surface diffusion of these two species is responsible for the spatial structures.

To show that general properties of promoters or poisons are sufficient to induce spontaneous reactive pattern formation on catalytic surfaces, we analyze a simple abstract model with three species $A, B$, and $C$. The first two species adsorb on the surface from the gas phase and react as $A+B \rightarrow P$, forming a product that immediately leaves

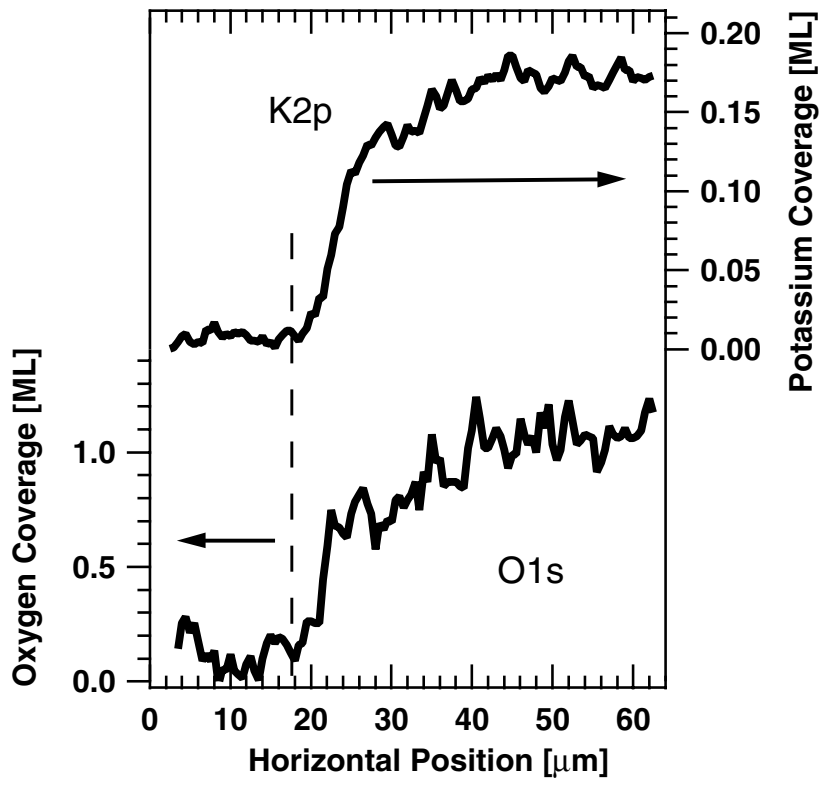

FIG. 2. Concentration profiles of potassium and oxygen in a stationary pattern obtained from in situ SPEM measurements of $\mathrm{O} 1 s$ and $\mathrm{K} 3 d$ core levels. Experimental conditions: $T=583 \mathrm{~K}, \quad p_{\mathrm{O}_{2}}=2 \times 10^{-7} \mathrm{mbar}, p_{\mathrm{H}_{2}}=0.76 \times 10^{-7} \mathrm{mbar}$, $\theta_{\mathrm{K}}=0.08$. the surface. The species $C$ represents an additional component, a catalytic promoter or poison, which is present on the surface but does not react with $A$ or $B$. However, it is characterized by strong attractive interactions with one of the reactive species $(B)$. Furthermore, we assume that surface mobility of $B$ is much lower than that of the species $A$ and $C$. In the mean-field approximation (see $[18-21])$, the system is described by a set of three kinetic equations for the local coverages $\theta_{A}, \theta_{B}$, and $\theta_{C}$,

$$
\begin{aligned}
\dot{\theta}_{A}= & k_{\mathrm{ads}}^{A} p_{A}\left(1-\theta_{A}\right)-k_{r} \theta_{A} \theta_{B}+D_{A} \nabla^{2} \theta_{A}, \\
\dot{\theta}_{B}= & k_{\mathrm{ads}}^{B} p_{B}\left(1-\theta_{B}\right)-k_{r} \theta_{A} \theta_{B}+D_{B} \nabla^{2} \theta_{B} \\
& +\nabla\left[\frac{D_{B}}{k_{B} T} \theta_{B}\left(1-\theta_{B}\right) \nabla U_{B}\right], \\
\dot{\theta}_{C}= & D_{C} \nabla^{2} \theta_{C}+\nabla\left[\frac{D_{C}}{k_{B} T} \theta_{C}\left(1-\theta_{C}\right) \nabla U_{C}\right] .
\end{aligned}
$$

Here $p_{A}$ and $p_{B}$ are partial pressures of species $A$ and $B$ in the gas phase, $k_{\mathrm{ads}}^{A}$ and $k_{\mathrm{ads}}^{B}$ are sticking coefficients, $k_{r}$ is the reaction rate constant, $D_{A}, D_{B}$, and $D_{C}$ are diffusion constants $\left(D_{B} \ll D_{A, C}\right)$, and $T$ is temperature. The last terms in Eqs. (2) and (3) describe viscous surface flows of adsorbates induced by potential gradients $\nabla U_{B}$ and $\nabla U_{C}$. The potentials are caused by attractive interactions between $B$ and $C$ and are given by $U_{B, C}(\mathbf{r})=-\int u\left(\mathbf{r}-\mathbf{r}^{\prime}\right) \theta_{C, B}\left(\mathbf{r}^{\prime}\right) d \mathbf{r}^{\prime}$, where $u(r)$ is the binary interaction potential between two adsorbed particles $B$ and $C$. For simplicity, it is chosen in the Gaussian form $u(r)=\left(u_{0} / \pi r_{0}^{2}\right) \exp \left(-r^{2} / r_{0}^{2}\right)$ where parameters $u_{0}$ and $r_{0}$ determine the strength and the radius of interactions. It is assumed that species $A, B$, and $C$ occupy different sublattices on the surface; for simplicity we also put $D_{A}=D_{C}=D$.

In the absence of reaction, strong attractive interactions between particles $B$ and $C$ lead to a phase transition, after which the system becomes separated into macroscopic regions occupied by the high- and low-density mixed phases. When reaction is added, periodic spatial patterns of alternating phase domains become formed. The characteristic wavelength of such domains is controlled by the reaction rate. The uniform stationary state of the system is characterized by coverages $\theta_{A}=a, \theta_{B}=b$, and $\theta_{C}=c$, where $a=(1+\gamma b)^{-1}$ and $b=(2 \gamma P)^{-1}(\gamma P-P-\gamma)+$ $\left[(\gamma P-P-\gamma)^{2}+4 \gamma P^{2}\right]^{1 / 2}$. We have introduced here the dimensionless reaction rate constant $\gamma=k_{r}\left(k_{\text {ads }}^{A} p_{A}\right)^{-1}$ and the parameter combination $P=\left(k_{\text {ads }}^{B} p_{B} / k_{\text {ads }}^{A} p_{A}\right)$. Testing the stability of this uniform state with respect to small nonuniform perturbations with different wave numbers $k$, we find that it becomes destabilized via a Turing bifurcation when the condition $\left(u_{0} / k_{B} T\right)^{2} \geq$ $[b(1-b) c(1-c)]^{-1}$ is satisfied. Note that, as follows from this condition, pattern formation is possible only if the interaction is strong enough and the promoter coverage exceeds a certain threshold. Moreover, when interaction strength is fixed, the patterns are found only at 
sufficiently high reaction rate constants; they also disappear for too strong reaction.

Nonlinear evolution of the patterns has been studied by numerical simulations. Figure 3(a) shows spatial profiles of coverages in the stationary nonuniform state obtained by numerical integration of Eqs. (1)-(3) for a one-dimensional system. We see that the adsorbates organize themselves in mixed clusters comprising $B$ and $C$ species. Spatial distributions of coverages $\theta_{B}$ and $\theta_{C}$ in the respective two-dimensional pattern are shown in Figs. 3(b) and 3(c). Note that, though such patterns arise from a Turing bifurcation, they are essentially different from the Turing patterns in reaction-diffusion systems. Energetic interactions between particles and the phase transition in the system are responsible for their emergence. The reaction rate constant $k_{r}$ in the considered simple model is independent on the promoter coverage, i.e., the promoter atoms have no direct effect on the reaction rate. Remarkably, the effective reaction rate becomes decreased even in absence of this effect when a periodic spatial pattern is formed on the catalytic surface. This is explained by the fact that the reaction proceeds
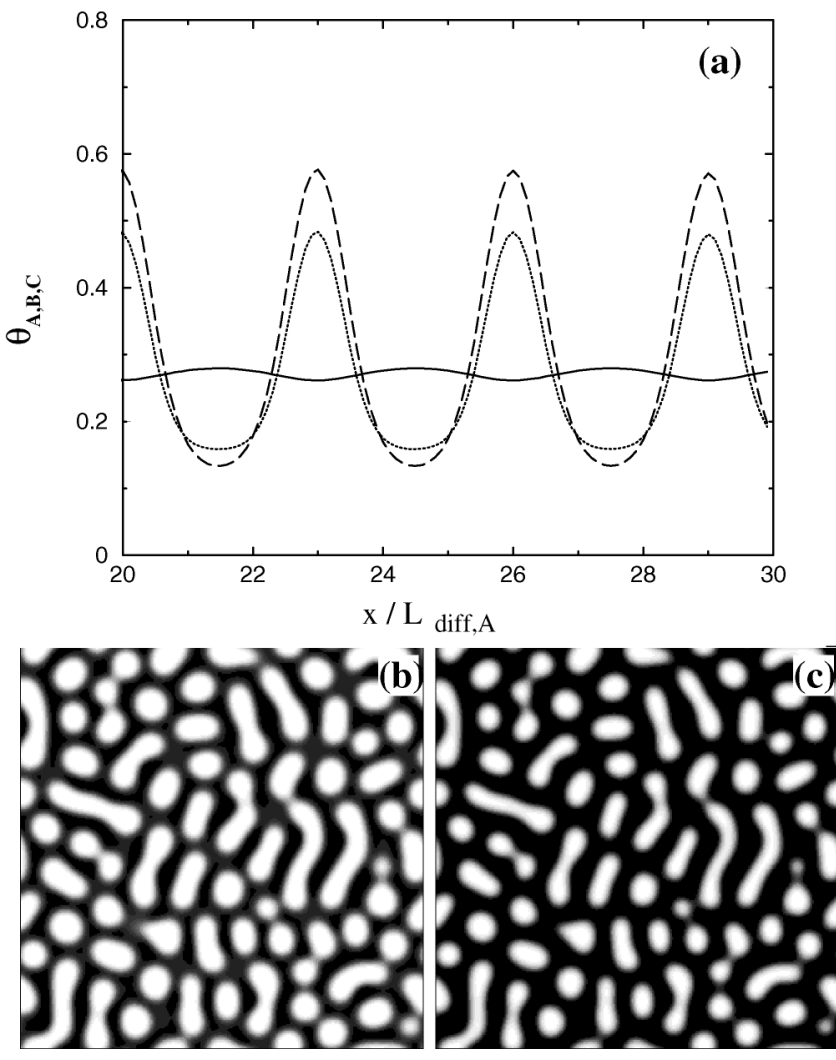

FIG. 3. Patterns in the general three-component model. (a) Asymptotic spatial profiles of coverages $\theta_{A}$ (dotted line), $\theta_{B}$ (solid line), and $\theta_{C}$ (dashed line) for $P=1, \gamma=10, c=$ $0.3, u_{0} k_{B} T=8, r_{0}=0.5 L_{\text {diff,A }}$, and $D_{B} D=0.1$. (b), (c) Snapshots of the distributions of coverages $\theta_{B}$ (b) and $\theta_{C}$ (c) in the two-dimensional simulation with the same parameters; white regions correspond to higher coverages. Periodic boundary and random initial conditions, the linear size is $30 L_{\text {diff,A }}$. less effectively when dense clusters are spontaneously built up.

Above, we have shown that stationary periodic patterns are already possible in a simple model and therefore can be generally expected. In the particular case of the $\mathrm{O}_{2}+\mathrm{H}_{2}$ reaction on $\mathrm{Rh}(110)$, a detailed realistic model can furthermore be formulated. It is based on the well established two-variable model for this bistable reaction, complemented by a third equation describing diffusion of potassium in a gradient of the chemical potential $[22,23]$ :

$$
\begin{aligned}
\dot{\theta}_{\mathrm{H}}= & \tilde{k}_{1} p_{\mathrm{H}_{2}}\left(1-\theta_{\mathrm{H}}-\beta \theta_{\mathrm{O}}\right)^{2}+\gamma \tilde{k}_{1} p_{\mathrm{H}_{2}}\left(1-\theta_{\mathrm{H}}\right)^{2} \\
& -k_{3} \theta_{\mathrm{H}}^{2}-2 k_{5} \theta_{\mathrm{O}} \theta_{\mathrm{H}}+D_{\mathrm{H}} \nabla^{2} \theta_{\mathrm{H}}, \\
\dot{\theta}_{\mathrm{O}}= & k_{2} p_{\mathrm{O}_{2}}\left(1-\theta_{\mathrm{H}}-\theta_{\mathrm{O}}\right)^{2}-k_{4} \theta_{\mathrm{O}}^{2}-k_{5} \theta_{\mathrm{O}} \theta_{\mathrm{H}} \\
& +D_{\mathrm{O}} \nabla^{2} \theta_{\mathrm{O}}, \\
\dot{\theta}_{\mathrm{K}}= & \nabla\left(D_{\mathrm{K}} \nabla \theta_{\mathrm{K}}\right)-\nabla\left[\frac{D_{\mathrm{K}}}{k_{B} T} \tilde{\theta}_{\mathrm{K}}\left(1-\tilde{\theta}_{\mathrm{K}}\right) E_{b} \nabla \theta_{\mathrm{O}}\right] .
\end{aligned}
$$

The first two equations contain the elementary steps of the Langmuir-Hinshelwood mechanism of the $\mathrm{O}_{2}+\mathrm{H}_{2}$ reaction, i.e., adsorption of $\mathrm{H}_{2}$ at regular $\left(\tilde{k}_{1}\right)$ and defect $\left(\gamma \tilde{k}_{1}\right)$ sites, desorption of $\mathrm{H}_{2}\left(k_{3}\right)$, oxygen adsorption $\left(k_{2}\right)$ and desorption $\left(k_{4}\right)$, diffusion of hydrogen $\left(D_{\mathrm{H}}\right)$ and oxygen $\left(D_{\mathrm{O}}\right)$, and the formation of water $\left(k_{5}\right)$. We assume that addition of the first proton to oxygen is rate limiting for the process of water formation. The third equation contains, in addition to regular Fickian diffusion, also a drift term that describes the surface flow of potassium atoms induced by their attractive interactions with adsorbed oxygen. Here $E_{b}$ is the gain in the adsoption energy as potassium moves from the reduced to the oxygencovered surface; moreover $\tilde{\theta}_{\mathrm{K}}=\theta_{\mathrm{K}} / \theta_{\mathrm{K}}^{\max }$, where $\theta_{\mathrm{K}}^{\max }=$ 0.22 is the maximum potassium coverage [24]. The rate constants are given in the Arrhenius form, $k_{i}=$ $\nu_{i} \exp \left(-E_{i} / k_{B} T\right)$, with $i=3,4,5$. A similar dependence holds for the diffusion constants, specifically for potassium diffusion, $D_{\mathrm{K}}=D_{\mathrm{K}}^{0} \exp \left(-E_{\mathrm{diff}, \mathrm{K}} / k_{B} T\right)$.

It is known that the potassium mobility on the oxygencovered surface is strongly reduced. This is taken into account in our realistic model by assuming a linear dependence of the activation energy for potassium diffusion on the oxygen coverage, $E_{\mathrm{diff}, \mathrm{K}}=E_{\mathrm{diff}, \mathrm{K}}^{\prime}+$ $\theta_{\mathrm{O}}\left(E_{\text {diff,K }}^{\prime \prime}-E_{\text {diff, } \mathrm{K}}^{\prime}\right)$ where $E_{\text {diff, } \mathrm{K}}^{\prime}$ and $E_{\text {diff,K }}^{\prime \prime}$ refer to the activation energies on the reduced and oxygen-covered surfaces, respectively. The experimentally observed decrease of the reactivity of oxygen in the presence of coadsorbed potassium is accounted in the model via the reduction of the hydrogen sticking coefficient in the presence of potassium, $\tilde{k}_{1}=k_{1} \exp \left(-\delta \theta_{\mathrm{K}}\right)$.

In numerical simulations of the realistic model, all constants are either taken from the experiment or fitted to experimental data [22]. In particular, the reduced reactivity of oxygen in the $\mathrm{K}+\mathrm{O}$ phase $(\delta)$, the attractive interaction between adsorbed potassium and oxygen atoms $\left(E_{b}\right)$, and the activation energy for potassium 


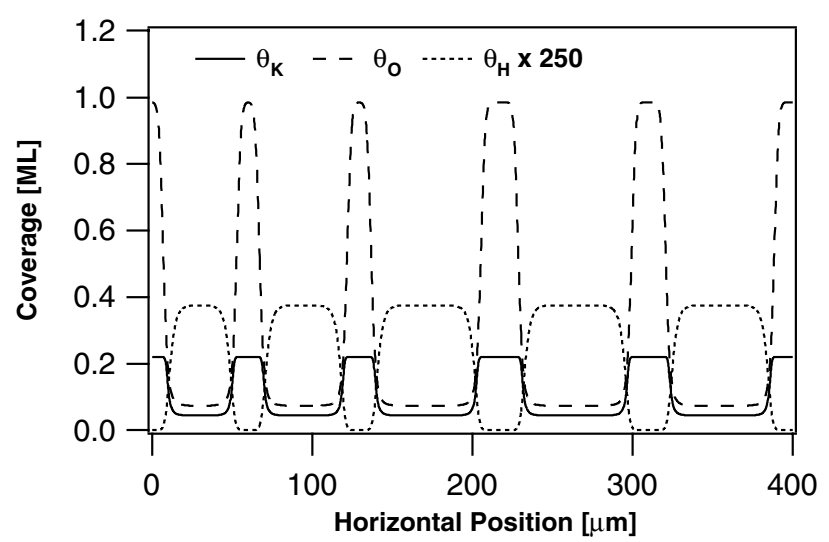

FIG. 4. Stationary pattern obtained by numerical simulations of the realistic model. Realistic 1D simulations showing the formation of a stationary pattern in $\mathrm{Rh}(110) / \mathrm{K} / \mathrm{O}_{2}+\mathrm{H}_{2}$. The model parameters are $p_{\mathrm{H}_{2}}=2.2 \times 10^{-6}$ mbar, $p_{\mathrm{O}_{2}}=$ $2.0 \times 10^{-6} \mathrm{mbar}, T=518 \mathrm{~K}$. Initial conditions: $\theta_{\mathrm{H}}=0.0$, $\theta_{\mathrm{O}}=0.2, \theta_{\mathrm{K}}=0.1$, with $20 \mu \mathrm{m}$ wide adsorbate free region in the center of a $1200 \mu \mathrm{m}$ wide interval (the figure shows a $400 \mu \mathrm{m}$ wide section). $T$-independent constants: $k_{1}=2.186 \times$ $10^{6} \mathrm{ML} \mathrm{s}^{-1} \mathrm{mbar}^{-1}, k_{2}=0.546 \times 10^{6} \mathrm{ML} \mathrm{s}^{-1} \mathrm{mbar}^{-1}, \beta=1.1$, $\gamma=4 \times 10^{-4}, \delta=6, E_{b}=0.2491 \times 10^{-22} \mathrm{~kJ} . T$-dependent constants: $\nu_{\mathrm{i}}\left[\mathrm{s}^{-1}\right], E_{\mathrm{i}}\left[10^{-22} \mathrm{~kJ}\right]$ preexponentials for diffusion in $\left[\mathrm{cm}^{2} \mathrm{~s}^{-1}\right]: k_{3}: 10^{13}, 1.1956 ; k_{4}: 10^{12}, 3.4041 ; k_{5}: 10^{13}, 1.4945$; $D_{\mathrm{H}}: 4 \times 10^{-3}, 0.2989 ; D_{\mathrm{O}}: 2.5 \times 10^{1}, 1.9926$; for $\mathrm{K}$ diffusion (see text): $D_{\mathrm{K}}^{0}=0.4 \mathrm{~cm}^{2} \mathrm{~s}^{-1}, E_{\text {diff, } \mathrm{K}}^{\prime}=0.6277 \times 10^{-22} \mathrm{~kJ}$, and $E_{\text {diff, } \mathrm{K}}^{\prime \prime}=1.4081 \times 10^{-22} \mathrm{~kJ}$.

diffusion on the oxygen-covered surface $\left(E_{\text {diff,K }}^{\prime \prime}\right)$ were all determined in the experiments $[15,16,22]$.

The simulations reveal that the uniform state of the system is unstable and a periodic spatial pattern of coverages spontaneously develops. Figure 4 gives an example of such pattern found in the one-dimensional simulations. Comparing it with Fig. 2, we notice that not only the profiles of coverages, but also the characteristic length scales of the experimental patterns are correctly reproduced in the realistic model. On the other hand, it is also similar to the stationary periodic patterns yielded by the abstract three-component model (Fig. 3). This indicates that the principal mechanisms, responsible for the formation of such structures, are already taken into account in this simple model.

In summary, we have shown that the stationary patterns found in the $\mathrm{O}_{2}+\mathrm{H}_{2}$ reaction on $\mathrm{Rh}(110)$ can be explained as corresponding to reactive phase separation. Our general theoretical analysis demonstrated that energetic interactions in connection with high mobility of the promoter/poison should typically suffice to generate such patterns. It can therefore be expected that effects of reactive phase separation should play an important role in a broad class of catalytic systems comprising promoters and poisons, as well as in the bimetallic systems with mobile components.
Financial support from the REACTOR programme of the European Science Foundation is acknowledged.

[1] S. C. Glotzer, E. A. Di Marzio, and M. Muthukumar, Phys. Rev. Lett. 74, 2034 (1995).

[2] M. Motoyama and T. Ohta, J. Phys. Soc. Jpn. 66, 2715 (1997).

[3] Q. Tran-Cong and A. Harada, Phys. Rev. Lett. 76, 1162 (1996).

[4] Y. Tabe and H. Yokoyama, Langmuir 11, 4609 (1995).

[5] R. Reigada, F. Sagues, and A. S. Mikhailov, Phys. Rev. Lett. 89, 038301 (2002).

[6] B. N. J. Persson, Surf. Sci. Rep. 15, 1 (1992)

[7] J. Verdasca, P. Borckmans, and G. Dewel, Phys. Rev. E 52, R4616 (1995).

[8] M. Hildebrand, A. S. Mikhailov, and G. Ertl, Phys. Rev. E 58, 5483 (1998).

[9] M. Hildebrand, A. S. Mikhailov, and G. Ertl, Phys. Rev. Lett. 81, 2602 (1998).

[10] M. Kiskinova, Poisoning and Promotion in Catalysis Based on Surface Science Concepts, in Studies in Surface Science and Catalysis 70, edited by B. Delmon and J. T. Yates (Elsevier, New York, 1992).

[11] R. D. Diehl and R. McGrath, Surf. Sci. Rep. 23, 43 (1996).

[12] F. Mertens and R. Imbihl, Chem. Phys. Lett. 242, 221 (1995).

[13] S. Günther, H. Marbach, R. Imbihl, A. Baraldi, S. Lizzit, and M. Kiskinova, J. Chem. Phys. 119, 12503 (2003).

[14] M. Kiskinova, E. Di Fabrizio, M. Gentili, and M. Marsi, Surf. Rev. Lett. 6, 265 (1999).

[15] H. Marbach, S. Günther, B. Luersen, L. Gregoratti, M. Kiskinova, and R. Imbihl, Catal. Lett. 83, 161 (2002).

[16] H. Marbach, S. Günther, L. Gregoratti, M. Kiskinova, and R. Imbihl, Chem. Phys. Lett. 364, 207 (2002).

[17] H. Marbach, G. Lilienkamp, H. Wei, S. Günther, Y. Suchorski, and R. Imbihl, Phys. Chem. Chem. Phys. 5, 2730 (2003).

[18] M. Hildebrand and A. S. Mikhailov, J. Phys. Chem. 100, 19089 (1996).

[19] M. Hildebrand and A. S. Mikhailov, J. Stat. Phys. 101, 599 (2000).

[20] D. G. Vlachos and M. A. Katsoulakis, Phys. Rev. Lett. 85, 3898 (2000).

[21] M. A. Katsoulakis and D. G. Vlachos, J. Chem. Phys. 119, 9412 (2003)

[22] M. Hinz, Doctor thesis, University of Hannover, 2003.

[23] A. Makeev and R. Imbihl, J. Chem. Phys. 113, 3854 (2000).

[24] A similar term, describing the flow of oxygen induced by its interactions with adsorbed potassium atoms, should generally be added to the equation describing the evolution of the oxygen coverage. Since the mobility of oxygen atoms is very low, inclusion of such a term does not, however, lead to any appreciable changes in the numerical results, as revealed by specially performed simulations. 